\title{
GERMINACIÓN Y SUPERVIVENCIA DE SEMILLAS DE THRINAX RADIATA (ARECACEAE), UNA ESPECIE AMENAZADA EN LA PENÍNSULA DE YUCATÁN
}

\author{
Erika Pérez, Gerardo Ceballos-González y luz María Calvo-Irabién ${ }^{1}$ \\ Centro de Investigación Científica de Yucatán, A.C. (CICY), Calle 43 No. 130, Col. Chuburná de Hidalgo, C.P. \\ 97200 Mérida, Yucatán, México. Tel 52 (999) 981-3923 ext. 370, Fax 52 (999) 981-3900. \\ 1 Autor para la correspondencia; correo-e: lumali@cicy.mx
}

\begin{abstract}
Resumen: Thrinax radiata ha sido catalogada como especie amenazada. La pérdida de su hábitat y el uso local son las principales amenazas. A través de experimentos de campo y laboratorio cuantificamos la germinación y supervivencia de semillas y evaluamos las principales causas de muerte. En laboratorio, más de 90\% de las semillas germinaron. La inmadurez del embrión disminuyó (70\%) y retardó la germinación. El almacenamiento de las semillas tuvo un efecto negativo sobre la germinación y la viabilidad de las semillas. En campo, únicamente un tercio de las semillas enterradas permanecieron viables después de seis meses; al año este porcentaje disminuyó a $0.1 \%$. Daños por insectos fueron la principal causa de muerte. El porcentaje acumulado de germinación fue de $15 \%$ después de 18 meses. Los datos sugieren que la disponibilidad de agua es el factor que limita la germinación en campo. Proponemos la siembra de esta palma en viveros comunitarios como una alternativa de manejo y conservación.
\end{abstract}

Palabras clave: almacenamiento, cloruro de tetrazolio, depredación, Quintana Roo, viabilidad.

\begin{abstract}
Thrinax radiata is listed as a threatened palm species in Mexico. Habitat loss and local use are the main threats. Analysis and comparison of field and laboratory experiments allowed us to obtain direct estimates of seed germination and survival, as well as an evaluation of the main causes of seed loss. Under controlled conditions germination was greater than $90 \%$. Embryo immaturity retarded and decreased (70\%) germination. Seed storage had a negative effect on seed viability. In the field, only one third of the seeds were viable after six months, decreasing to $0.1 \%$ a year later. Insect damage was the main cause of death. Field percentage of cummulative germination was $15 \%$ after 18 months; insufficient water is suggested as the factor delaying germination. Propagation of this palm in community-owned nurseries is being implemented as an in situ conservation and management alternative. This practice has smaller demographic impact than trunk harvest.
\end{abstract}

Key words: Quintana Roo, seed predation, seed storage, seed viability, tetrazolium chloride.

as especies de la familia Arecaceae, comúnmente cono- cidas como palmas, son de gran importancia tanto ecológica como económica. Las palmas son un componente importante en la mayoría de los bosques tropicales y constituyen un recurso clave para la fauna, especialmente en períodos de escasez de alimento (Terborgh, 1986; Gentry, 1990; Scariot, 1999). Aunado a lo anterior, las especies de palmas son utilizadas por el hombre en una gran diversidad de actividades (Balick y Beck, 1990).

En la Península de Yucatán existen 20 especies de palmas silvestres (Orellana y Durán, 1992; Quero 1992a, b;
Sánchez-Sánchez e Islebe, 2002). Actualmente, la Península de Yucatán presenta aceleradas tasas de deforestación, pues entre 0.2 y $4.5 \%$ de la superficie boscosa se pierde anualmente en función de los patrones de ocupación humana y de los esquemas de desarrollo socioeconómico (CortinaVillar et al., 1999; Turner et al., 2001).

Thrinax radiata Schult. et Schult.f. es una especie de palma de importancia económica y cultural entre los mayas peninsulares (Standley, 1930; Roys, 1931; Olmsted y Álvarez-Buylla, 1995; La Torre-Cuadros e Islebe, 2003). Desde 1994 esta especie está catalogada oficialmente como 
amenazada en territorio mexicano en la Norma Oficial Mexicana NOM-ECOL 59-2001 (DOF, 2002). El mayor riesgo que enfrentan sus poblaciones es la destrucción de la selva mediana y la vegetación costera, comunidades que constituyen su hábitat natural; otra presión es el uso local de los troncos como material de construcción (Olmsted y Álvarez-Buylla, 1995). En la Península de Yucatán el corte y la venta ilegal de esta especie son frecuentes y carecen de un control adecuado (Calvo-Irabién y Ceballos-González, 2004). Como consecuencia de este escenario, cualquier explotación comercial de esta palma necesita de una autorización basada en un plan de manejo (DOF, 2002).

El entendimiento de los factores que afectan el tamaño de las poblaciones en condiciones naturales nos permite desarrollar estrategias para su conservación y utilización (Schemske et al., 1994). La importancia de las semillas en la persistencia de las poblaciones, así como para su dispersión, establecimiento y diversidad genética ha sido descrita en detalle (Harper, 1977; Fenner, 1985; Murdoch y Ellis, 1992; Vázquez-Yanes y Orozco-Segovia, 1993; Bewley y Black, 1994). Por tanto, el conocimiento de la biología de las semillas es una herramienta básica para el manejo y la conservación de las poblaciones silvestres (Vázquez-Yanes y Orozco-Segovia, 1993). Sin embargo, aun cuando se reconoce que la germinación de semillas y la supervivencia de las plántulas son una parte crucial del ciclo de vida de las plantas y de su dinámica poblacional, el destino de las semillas en el suelo es un tema poco estudiado, especialmente en el caso de las especies de palmas (OrozcoSegovia et al., 2003).

Dada su importancia como plantas ornamentales, la germinación en semillas de palmas ha sido documentada ampliamente (Brown, 1976; Broschat y Donselman, 1986, 1987; Carpenter y Gilman, 1988; Marcus y Banks, 1999; Meerow, 2000). En estudios previos, para diversas especies de palmas se ha considerado que son varios los factores que determinan en mayor medida su respuesta germinativa: la inmadurez del embrión, la cubierta de la semilla, las condiciones de luz, así como el manejo al que se someten las semillas (Orozco-Segovia et al., 2003).

Únicamente para 102 especies de palmas -aproximadamente el 5\% de la familia-, se tiene información detallada sobre las condiciones adecuadas de almacenamiento. De esta fracción, sólo una cuarta parte presenta semillas que permanecen viables bajo condiciones convencionales de almacenamiento (Wood y Pritchard, 2003). El entendimiento del proceso de germinación bajo condiciones controladas permite, en parte, explicar los patrones de abundancia y distribución de especies vegetales que observamos en la naturaleza. Asimismo, estos estudios complementan la información obtenida en experimentos de campo.

El objetivo de este estudio fue analizar y comparar la germinación y la supervivencia de las semillas de Thrinax radiata bajo condiciones naturales y controladas. Basados en la información generada, se presentan sugerencias de manejo y conservación para esta especie.

Este estudio forma parte de un proyecto, solicitado por las autoridades de tres ejidos ubicados en el NO de Quintana Roo, cuyo objetivo es la obtención de un permiso de aprovechamiento de Thrinax radiata. Dicho permiso deberá estar basado en un plan de manejo que defina las condiciones del aprovechamiento y su impacto sobre las poblaciones manejadas (Calvo-Irabién et al., 1998; CalvoIrabién y Ceballos-González, 2004). En los bosques de los ejidos Kantunilkin, Solferino y Chiquilá, Thrinax radiata es una de las especies dominantes del estrato arbóreo con valor económico y cultural para los pobladores de dichos ejidos (La Torre-Cuadros e Islebe, 2003).

\section{Materiales y métodos}

Especie de estudio. Thrinax radiata, localmente conocida por su nombre en maya chit, presenta una distribución caribeña. En México es la única especie del género y se distribuye únicamente en la Península de Yucatán (Quero, 1992a, b). El chit es una palma solitaria con un tallo delgado, recto, que en ocasiones alcanza alturas de hasta $18 \mathrm{~m}$. El crecimiento de los individuos es lento, dependiendo principalmente de las condiciones de luz en las que se desarrollen. La edad estimada para una palma de $15 \mathrm{~m}$ de altura oscila entre 100 y 145 años (Olmsted y ÁlvarezBuylla, 1995). La especie presenta hojas en forma de abanico de hasta $1 \mathrm{~m}$ de diámetro. La inflorescencia es ramificada, de entre 50 y $60 \mathrm{~cm}$ de longitud y presenta flores hermafroditas, pequeñas $(c a .5 \mathrm{~mm})$, que varían en número pero pueden llegar hasta 15,000 (G. Ceballos-González, no publicado). Los individuos de chit comienzan a reproducirse cuando el tronco alcanza $3 \mathrm{~m}$ de altura y producen entre 300 y 3,000 semillas (Olmsted y Álvarez-Buylla, 1995; Calvo-Irabién y Ceballos-González, 2004). En el área de estudio la fructificación se presenta de junio a noviembre; los frutos son dispersados por animales, principalmente mamíferos (G. Ceballos-González, obs. pers.). Los frutos son de color verde cuando inmaduros y blanco al madurar (Quero, 1992a, b). La semilla tiene un diámetro promedio de $7 \mathrm{~mm}$, es color café, lisa y brillante. Cada fruto porta una sola semilla con un embrión subapical (Quero, 1992b).

El uso del chit es antiguo entre los mayas (Standley, 1930; Roys, 1931). No obstante, desde hace aproximadamente 30 años su uso se ha intensificado especialmente en las costas, en donde los troncos son apreciados para la construcción de trampas de langosta (Cabrera et al., 1982) y para el acabado de paredes en hoteles y restaurantes (Cabrera et al., 1982; Quero, 1992). Otros usos de menor impacto son la cosecha de las hojas para el techado de las casas mayas tradicionales y de palapas turísticas (Flores y 
Espejel, 1994), así como para la fabricación de escobas y sombreros (Flores, 2001). De igual manera, hay reportes del uso de diferentes partes de la planta con fines medicinales, artesanales y culinarios (Almanza, 2000; CalvoIrabién y Ceballos-González, 2004). En la actualidad el chit es apreciado como planta de ornato, tanto de interiores como de exteriores (Durán et al., 2000).

Sitio de estudio. El trabajo de campo se llevó a cabo en bosques de los ejidos Kantunilkin, Solferino y Chiquilá, pertencientes al municipio Lázaro Cárdenas, en el estado de Quintana Roo $\left(21^{\circ} 12^{\prime}-21^{\circ} 25^{\prime}\right.$ N, $87^{\circ} 06^{\prime}-87^{\circ} 30^{\prime}$ O; figura 1). Los ejidos se encuentran en la zona de amortiguamiento del Área Natural Protegida Yum Balam, decretada el 6 de junio de 1994 (DOF, 1994). La vegetación predominante en esta zona ha sido clasificada como selva mediana subperennifolia (Olmsted et al., 2000), con una altura del dosel entre 12 y 17 m. Las especies arbóreas más abundantes son Manilkara sapota, Simarouba glauca, Swartzia cubensis, Vitex gaumeri, Metopium brownei, Bursera simaruba, Sabal yapa y Thrinax radiata (Olmsted et al., 2000; La Torre-Cuadros e Islebe, 2003).

El clima de la región es cálido subhúmedo. La temperatura media anual es de $25.8^{\circ} \mathrm{C}$ y la precipitación anual oscila entre 700 y $1,400 \mathrm{~mm}$, presentando un gradiente decreciente de sur a norte (de Kantunilkin a Chiquilá). En la zona se observa una época de secas entre los meses de noviembre a mayo (Orellana et al., 1999). Los suelos son jóvenes, someros y rocosos, con poco contenido de materia orgánica (Lazcano et al., 1995).

Los pobladores de esta zona son de origen maya, la mayoría provenientes de Yucatán, aunque también existe un grupo pequeño de pobladores de origen totonaco, migrantes de Veracruz. Las principales actividades productivas son la agricultura de temporal y la extracción de productos forestales, tanto maderables como no maderables. La ganadería, la pesca y la apicultura son actividades complementarias (La Torre-Cuadros e Islebe, 2003).

Germinación y almacenamiento de semillas bajo condiciones controladas. En los bosques de Kantunilkin y Solferino se colectaron frutos, tanto inmaduros (color verde) como maduros (color blanco), provenientes de 13 individuos reproductivos de Thrinax radiata. Los frutos fueron transportados al laboratorio en bolsas de plástico perforadas; una vez en el laboratorio la pulpa fue removida a mano y las semillas se limpiaron y se lavaron con agua. El contenido de humedad de las semillas recién colectadas se cuantificó en una muestra de 15 semillas maduras. Para ello, las semillas limpias fueron partidas por la mitad con una navaja, se separó el embrión del endospermo y se pesaron ambos de manera independiente en una balanza analítica (Sartorius, $0.0001 \mathrm{~g}$ ). Posteriormente el embrión y el endospermo se secaron en un horno a $103 \pm 2{ }^{\circ} \mathrm{C}$, hasta

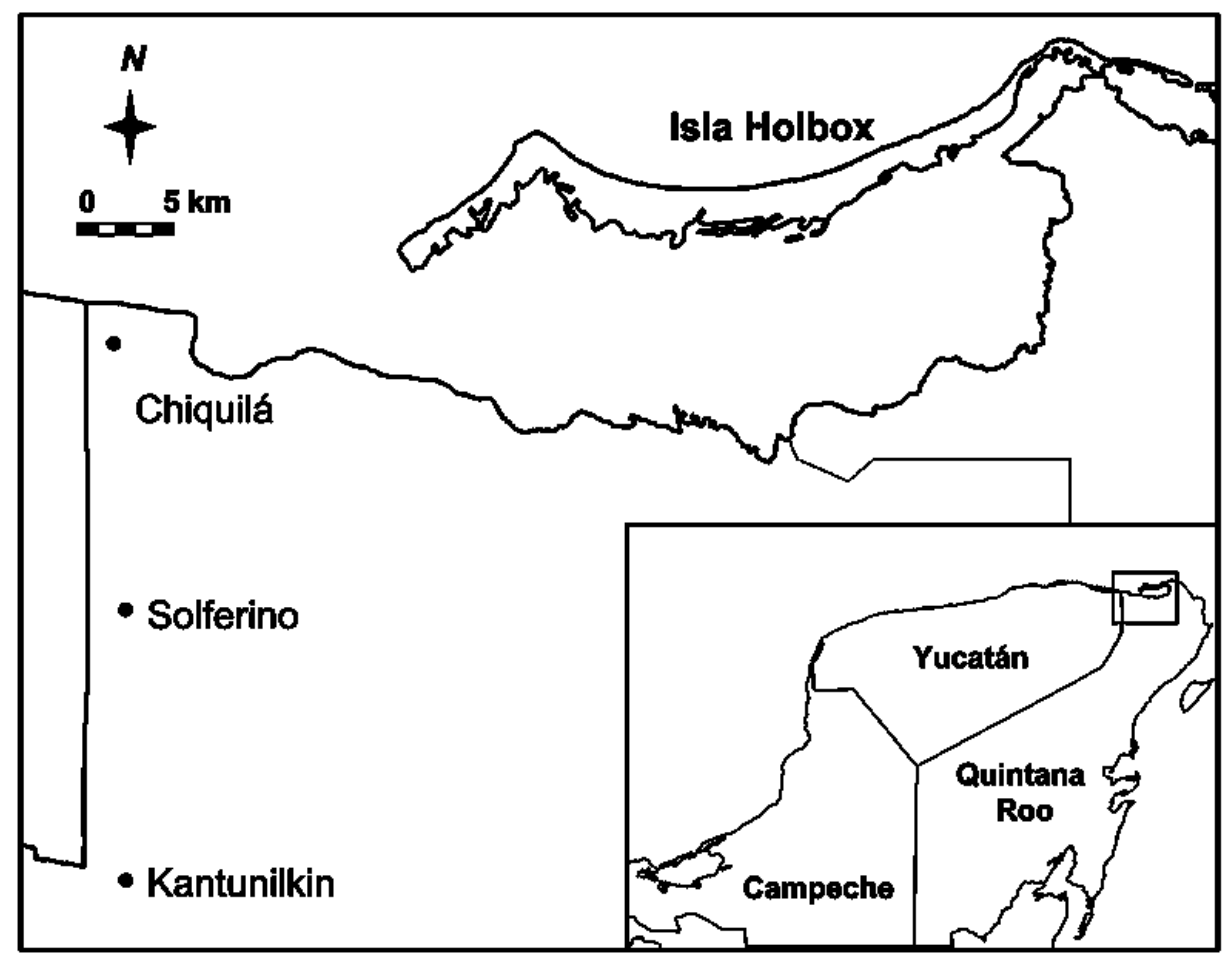

Figura 1. Mapa de la localidad donde se muestra la ubicación de los tres sitios permanentes de estudio. 
alcanzar el peso constante, siguiendo el protocolo recomendado por ISTA (1999). Una vez secos, se registró el peso del embrión y el del endospermo.

Los métodos generales utilizados para evaluar las dos variables de respuesta, porcentaje de germinación y porcentaje de viabilidad, analizadas en el presente estudio se describen a continuación. Para el porcentaje de germinación, las semillas colectadas en campo, una vez limpias, se sembraron en charolas de germinación (recipientes cerrados, de plástico transparente y $\left.10 \mathrm{~cm}^{2}\right)$, utilizando como sustrato tierra (k'ankab)-arena (T/A) en una proporción 2:1 (Durán et al., 2000). El sustrato de los recipientes de germinación fue regado frecuentemente con la finalidad de mantenerlo húmedo. Se sembraron un total de 30 semillas por charola, a $0.5 \mathrm{~cm}$ de profundidad, conformando lotes experimentales de 10 recipientes (repeticiones) cada uno. Cada lote experimental (300 semillas) fue asignado a uno de seis tratamientos diferentes. Los tratamientos aplicados fueron los siguientes. (1) Control: semillas provenientes de frutos maduros. (2) Inmadurez: semillas provenientes de frutos inmaduros. (3) Escarificación mecánica: semillas maduras cuya testa fue reducida con una lima de metal en la cálaza para garantizar no dañar al embrión (Quero, 1992b); la testa de la semilla se lijó hasta observar el color blanquecino del endospermo. (4) Oscuridad: semillas maduras, sembradas en recipientes de plástico cubiertos con papel aluminio. (5) Altas temperaturas: semillas maduras que, antes de su siembra, fueron sometidas a temperaturas de 80 y $100^{\circ} \mathrm{C}$ durante $5 \mathrm{~min}$. (6) Tiempo-temperatura de almacenamiento: se colocaron 2,400 semillas, maduras y limpias, en 20 recipientes de plástico transparente $(275 \mathrm{ml})$ herméticamente cerrados, en los que las semillas ocupaban $c a$. $20 \%$ del volumen. Las semillas así almacenadas fueron colocadas en dos condiciones contrastantes de temperatura; ambiente $\left(27 \pm 0.31^{\circ} \mathrm{C}\right.$ y 59 $\pm 1.0 \% \mathrm{RH})$ y cuarto frío $\left(8.2 \pm 0.1{ }^{\circ} \mathrm{C}\right.$ y $\left.62 \pm 0.1 \% \mathrm{RH}\right)$. Después de 1, 3, 6 y 12 meses de almacenamiento se cuantificó el porcentaje de germinación y el porcentaje de semillas viables. Las charolas de germinación fueron colocadas en un cuarto bajo condiciones controladas de temperatura $\left(27 \pm 0.31^{\circ} \mathrm{C}\right)$, humedad relativa (RH $\left.59 \pm 1.0 \%\right)$ y fotoperíodo (12 horas) en el Centro de Investigación Científica de Yucatán (CICY). El registro de las semillas germinadas se hizo en un cuarto oscuro con luz verde, cada segundo día, durante 3.5 meses. Se consideró una semilla como germinada cuando se observó la emergencia de la radícula; una vez registrada la semilla se desechaban para evitar confusiones.

En el caso del porcentaje de viabilidad, éste se cuantificó por medio de una prueba de tinción utilizando cloruro de 2 , 3, 5 trifeniltetrazolio (TZ) siguiendo el protocolo descrito en Yacubson (1980) y Moreno (1996). En los tratamientos control, inmadurez, oscuridad, escarificación y altas temperaturas, la viabilidad de las semillas se evaluó única- mente en aquellas semillas no germinadas después de 3.5 meses en el cuarto de germinación; por lo tanto, el número de semillas en cada una de las 10 repeticiones fue variable. En el caso del tratamiento tiempo-temperatura de almacenamiento el porcentaje de viabilidad se evaluó en 10 repeticiones de 30 semillas cada una.

Adicionalmente, y con el objetivo de determinar el porcentaje de viabilidad inicial de las semillas maduras e inmaduras colectadas, se aplicó la prueba de TZ a 300 semillas, repartidas en 10 lotes de 30 semillas cada uno.

Germinación y supervivencia de semillas en condiciones naturales. El destino de las semillas en el suelo fue evaluado en los bosques de los tres ejidos, Kantunilkin, Solferino y Chiquilá, donde se estableció un cuadro permanente $\left(2,000 \mathrm{~m}^{2}\right)$ en cada sitio para el estudio demográfico del chit. La distancia aproximada entre sitios fue de $15 \mathrm{~km}$ (figura 1). Las semillas utilizadas en el experimento se obtuvieron de 10 individuos reproductivos de Thrinax radiat $a$ ubicados en la periferia de los cuadros permanentes; de estos individuos se colectaron frutos maduros (blancos) los primeros días de noviembre. Las semillas se limpiaron utilizando el mismo procedimiento descrito previamente para las semillas utilizadas en los experimentos de laboratorio.

En este experimento de campo se utilizaron charolas abiertas de plástico $(30 \times 30 \mathrm{~cm})$ con 36 celdas en cada charola. Las charolas fueron llenadas con tierra de la región, previamente pasada por un tamiz de $2 \mathrm{~mm}$ de apertura de malla para asegurar que no contuviera semillas de chit. Posteriormente se sembraron 36 semillas, maduras y limpias, una en cada una de las 36 celdas de la charola, a una profundidad de $0.5 \mathrm{~cm}$. En cada uno de los tres cuadros permanentes se colocaron 30 charolas en el suelo, de tal manera que la parte superior de la charola quedara a ras del suelo. A los 6, 12 y 18 meses (nov 1999-may 2000, nov 1998-nov 1999, nov 1998-may 2000, respectivamente) 10 charolas fueron desenterradas de cada sitio (30 en total) y fueron llevadas al laboratorio donde la tierra de cada celda se revisó cuidadosamente para registrar la presencia de semillas, o sus restos. En el cuadro 1 se describen las categorías en las que fueron clasificadas las semillas recuperadas.

Las charolas que contenían las semillas de chit fueron colocadas a finales de noviembre, una vez que la dispersión natural de semillas había terminado, para evitar así la entrada de nuevas semillas a las charolas experimentales. Una vez colocadas las charolas, el conteo de la germinación (presencia de radícula o plántula) se realizó cada 15 días; la semilla registrada como germinada se eliminaba de la charola para evitar registrarla de nuevo.

Análisis de datos. El efecto de cuatro tratamientos (control, inmadurez, oscuridad y escarificación) sobre el porcentaje de germinación se evaluó estadísticamente utilizando un Análisis de Varianza (ANDEVA) de una vía, mientras que 
Cuadro 1. Categorías de clasificación de las semillas de Thrinax radiata encontradas en el suelo de las charolas experimentales (ver texto).

\begin{tabular}{cl}
\hline Categoría & \multicolumn{1}{c}{ Descripción } \\
\hline Perdidas & $\begin{array}{l}\text { Semillas que, en el laboratorio, no se encontraron } \\
\text { en el suelo de las celdas de las charolas experimen- } \\
\text { tales. }\end{array}$ \\
Viables & $\begin{array}{l}\text { Semillas encontradas en las celdas, de consistencia } \\
\text { dura y apariencia sana, a las que se aplicó la prue- } \\
\text { ba de tinción con TZ y en las que el embrión com- } \\
\text { pleto se tiñó de rojo vivo e intenso de forma homo- } \\
\text { génea, teñido en 3/4 partes y/o coloración rosada. }\end{array}$ \\
Muertas & $\begin{array}{l}\text { Semillas encontradas en las celdas, suaves al tacto, } \\
\text { podridas, necrosadas, picadas por insectos, o con } \\
\text { hongos. También se incluyen las semillas duras al } \\
\text { tacto pero que al teñirlas con TZ, el embrión per- } \\
\text { maneció blanco, o parcialmente teñido. }\end{array}$ \\
Germinadas & $\begin{array}{l}\text { Semillas cuya radícula y/o plántula emergió en el } \\
\text { campo y se registró como germinada, o bien presen- } \\
\text { taron la radícula emergida cuando se revisaron las } \\
\text { charolas en el laboratorio. }\end{array}$ \\
\hline
\end{tabular}

en el caso del efecto del tiempo y temperatura de almacenamiento sobre los porcentajes de germinación y viabilidad se utilizó una prueba de ANDEVA de dos vías. Esta misma prueba estadística fue empleada para evaluar el efecto de la variación espacial (sitio) y temporal (6, 12 y 18 meses) sobre el porcentaje de germinacion de las semillas en el experimento de campo. En todos los análisis estadísticos a los datos de porcentaje de germinación y de viabilidad se les aplicó una transformación arco-seno para cumplir con los supuestos del ANDEVA (Sokal y Rohlf, 1995). Posteriormente se realizaron pruebas de Tukey para la comparación múltiple de medias (Sokal y Rohlf, 1995). En todos los casos se presentan los valores promedio \pm su error estándar.

Con el propósito de explorar la relación entre la cobertura del dosel y el porcentaje de germinación en condiciones naturales, se utilizó un densiómetro convexo (convex spherical crown densiometer; Forestry Suppliers Inc.) para estimar el porcentaje de apertura del dosel encima de las charolas de germinación. Las medidas se realizaron colocando el densiómetro $50 \mathrm{~cm}$ encima de la charola de germinación y contando el número de puntos sin cobertura vegetal en el densiómetro (Lemmon, 1956). Para evaluar la correlación entre las variables apertura del dosel y porcentaje de germinación se utilizó una correlación de rangos de Spearman (Sokal y Rohlf, 1995).

\section{Resultados}

Germinación y almacenamiento de semillas bajo condiciones controladas. El contenido de humedad de las semillas al momento de la cosecha fue de $46.7 \pm 7.5 \%$ para el embrión y $28.4 \pm 0.7 \%$ para el resto de la semilla. Las pruebas de viabilidad no mostraron diferencias en la viabilidad inicial de semillas maduras e inmaduras $(99.0 \pm 0.7 \%$ y $98.7 \pm 0.7 \%$, respectivamente). En contraste, después de 3.5 meses en el cuarto de germinación, únicamente permanecieron viables aquellas semillas provenientes de frutos inmaduros, con $28.3 \%$. Los tratamientos control, escarificación y oscuridad no presentaron semillas viables al finalizar el experimento.

El porcentaje de semillas que germinaron después de 3.5

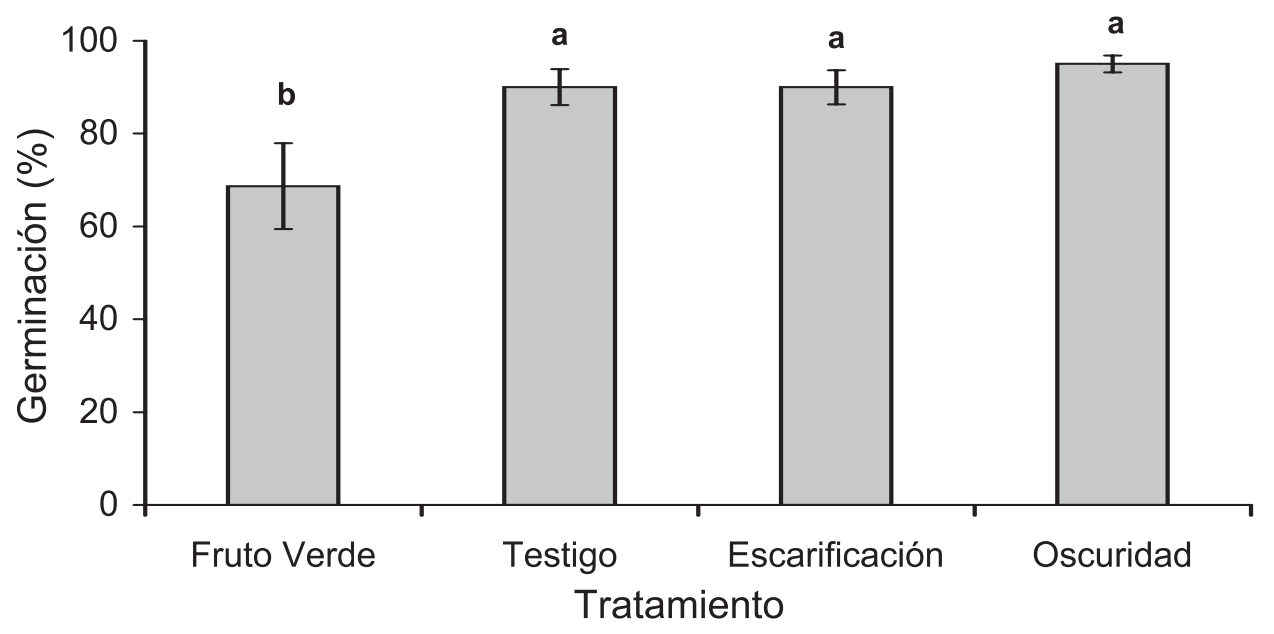

Figura 2. Porcentaje de germinación, bajo condiciones controladas $\left(27^{\circ} \mathrm{C}, 59 \%\right.$ humedad relativa y 12 horas de fotoperíodo), de semillas de Thrinax radiata sometidas a diferentes tratamientos. Se presentan los valores promedio y el error estándar. Las barras señaladas con la misma letra no mostraron diferencias estadísticamente significativas. 
meses en condiciones controladas se vio negativamente afectado por el tratamiento de alta temperatura. Ninguna de las semillas bajo este tratamiento germinó y la viabilidad posterior al tratamiento fue de $2.0 \pm 2.0 \%$ y $6.0 \pm 6.0 \%$, para 80 y $110^{\circ} \mathrm{C}$, respectivamente.

En las semillas provenientes de frutos maduros sometidas a los tratamientos control, oscuridad y escarificación, el porcentaje de germinación fue igual o mayor que $90 \%$ (figura 2). En el caso de las semillas provenientes de frutos inmaduros, el porcentaje promedio de germinación fue significativamente menor $\left(F_{3,36}=4.1, P=0.013\right.$; figura 2$)$; únicamente $70 \%$ de las semillas germinaron bajo este tratamiento. Asimismo, el tiempo de inicio de la germinación fue mayor en semillas inmaduras (27.0 4.0 días $)$, mientras que las semillas maduras colocadas en oscuridad fueron las que germinaron más rápidamente $(13.7 \pm 0.4$ días después de la siembra).

El ANDEVA para evaluar el efecto de la temperatura y el tiempo de almacenamiento sobre la germinación de las semillas mostró una interacción estadísticamente significativa $\left(F_{3,72}=10.2, P \leq 0.0001\right)$. Igualmente, el efecto principal de ambos factores fue significativo $\left(F_{1,72}=14.7, P \leq\right.$ 0.0003 para temperatura y $F_{3,72}=161.5, P \leq 0.0001$, para tiempo de almacenamiento; figura 3). El porcentaje de germinacion disminuyó al transcurrir el tiempo de almacenamiento; el efecto fue más evidente después de 3 meses. El porcentaje de germinación de semillas almacenadas por un año disminuyó a $9.3 \pm 1.6 \%$ a temperatura ambiente y a $4.0 \pm 0.6 \%$ en el cuarto frío (figura $3 \mathrm{a}$ ).

El efecto del tiempo y de las condiciones de almacenamiento sobre la viabilidad de las semillas fue semejante al observado para la germinación. La viabilidad de las semillas disminuyó considerablemente después de 6 meses de almacenamiento y al año; únicamente 16 y $21 \%$ de las semillas almacenadas a temperatura ambiente y en cuarto frío, respectivamente, permaneció viable (figura 3b). El ANDEVA mostró una interacción significativa de los factores tiempo y temperatura de almacenamiento $\left(F_{3,70}=\right.$
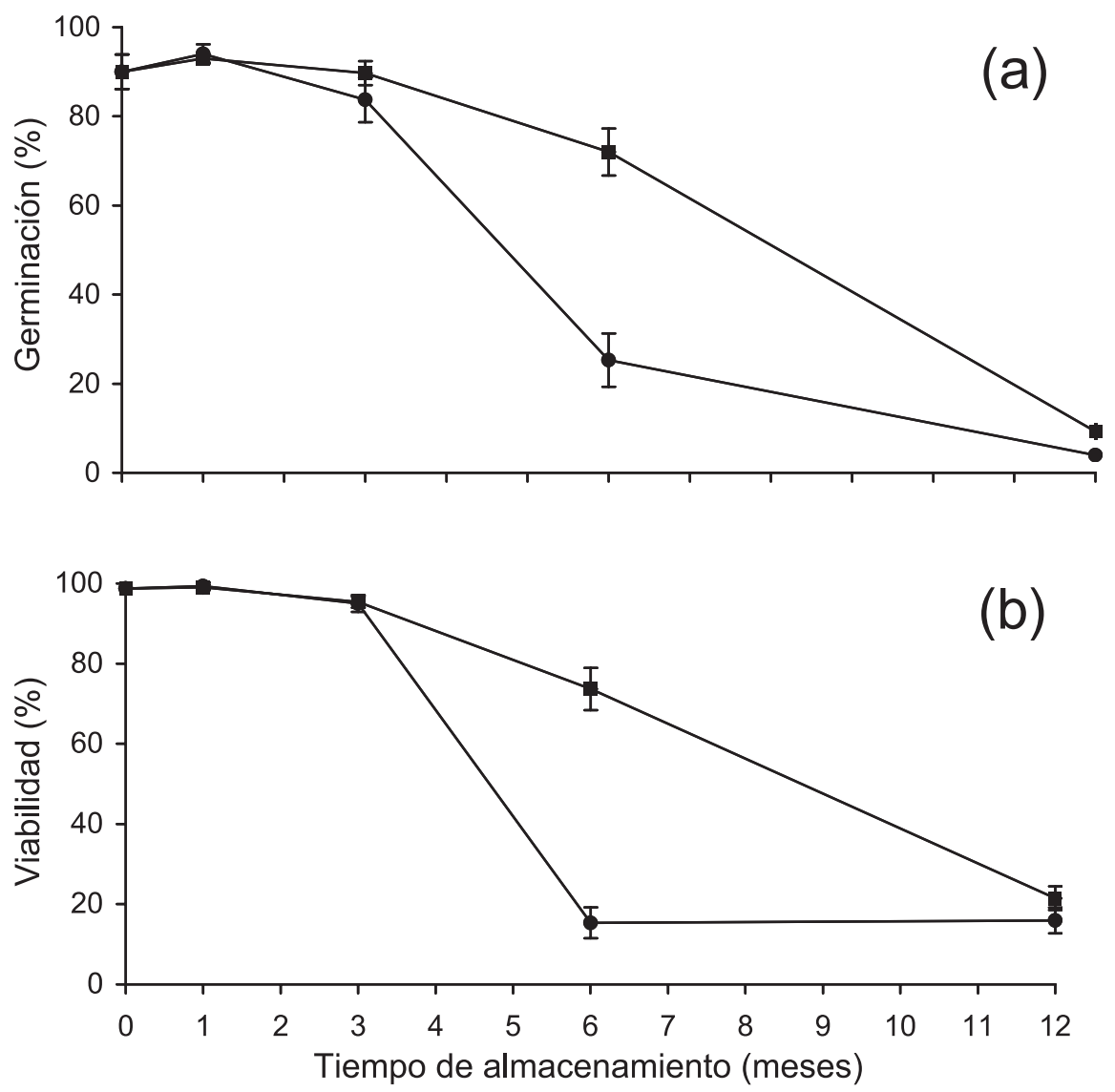

Figura 3. Efecto del tiempo y de la temperatura de almacenamiento en el porcentaje de germinación (a) y la viabilidad (b) de semillas de Thrinax radiata. La germinación se registró bajo condiciones controladas $\left(27^{\circ} \mathrm{C}, 59 \%\right.$ humedad relativa y 12 horas de fotoperíodo). La viabilidad de semillas se detectó con una prueba de tinción de cloruro de tetrazolio. $\mathbf{\square}=$ semillas almacenadas a temperatura ambiente $\left(27^{\circ} \mathrm{C}\right) ; \boldsymbol{\top}=$ semillas almacenadas en cuarto frío $\left(8^{\circ} \mathrm{C}\right)$. Se presentan los valores promedio con su error estándar. 

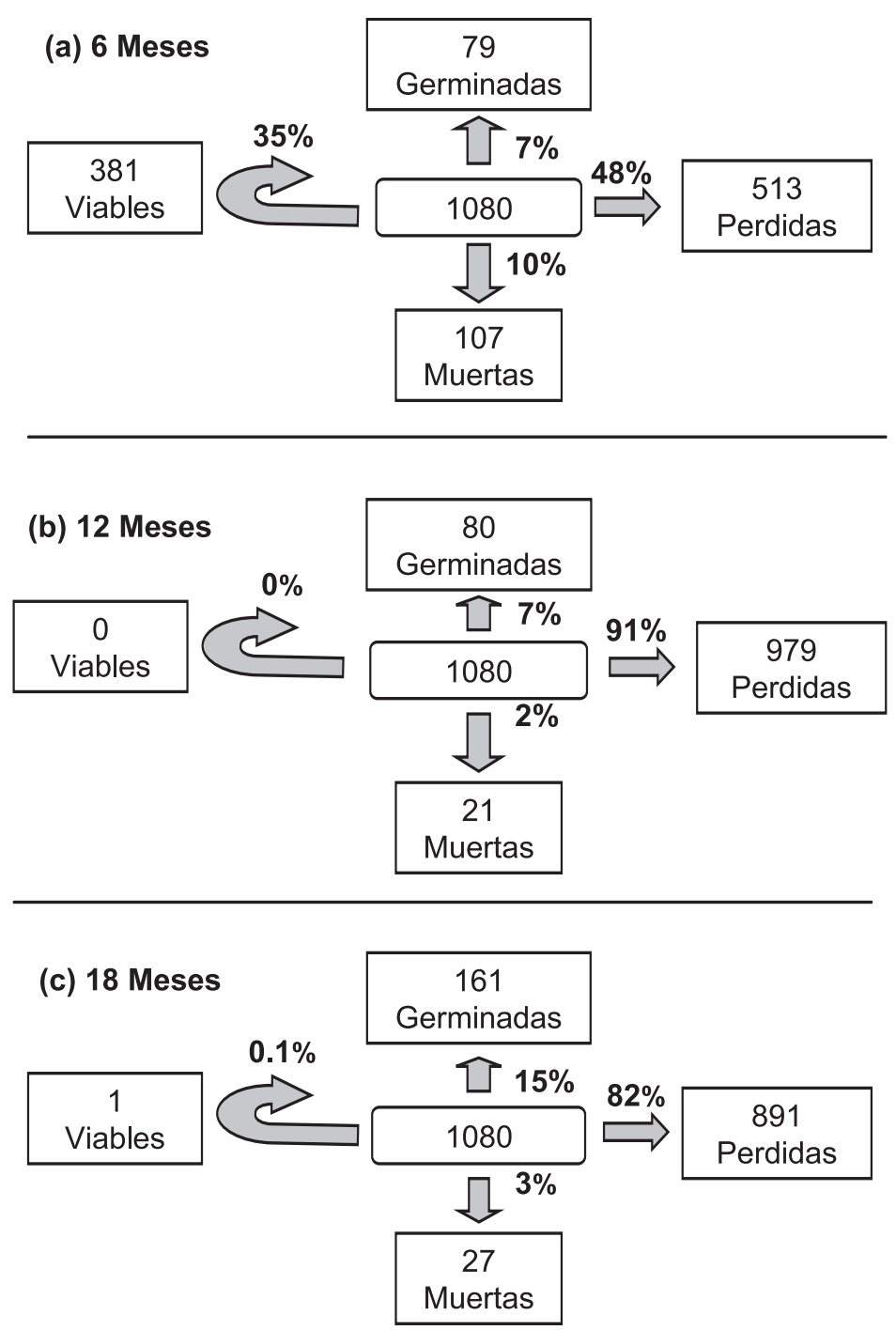

Figura 4. Destino de las semillas de Thrinax radiata enterradas durante 6, 12 y 18 meses. Las cifras dentro de los rectángulos representan el número de semillas en cada categoría; las cifras asociadas a las flechas muestran el porcentaje de semillas. Se presentan los datos de los tres sitios de manera conjunta.

$17.9, P \leq 0.001)$, así como de dichos factores por separado $\left(F_{1,70}=21.7, P \leq 0.001\right.$ y $F_{3,70}=137.1, P \leq 0.0001$, para temperatura y tiempo, respectivamente).

Germinación y supervivencia de semillas en condiciones naturales. El porcentaje acumulado de germinación a los 6 y 12 meses de sembradas las semillas presentó un valor de $7.3( \pm 1.42 \%)$ y $7.4( \pm 1.6 \%$; figura $4 \mathrm{a}, \mathrm{b})$, respectivamente. Este porcentaje fue mayor a los 18 meses $(14.9 \pm 2.22 \%$; figura 4c). El ANDEVA mostró un efecto significativo del efecto del tiempo de permanencia en el suelo $\left(F_{2,81}=5.89\right.$, $P=0.004)$, sobre el porcentaje de semillas germinadas. De igual manera, el efecto del factor sitio sobre el porcentaje de germinación también resultó significativo $\left(F_{2,81}=4.47\right.$, $P=0.014)$, pero no la interacción de ambos factores $\left(F_{4,81}\right.$ $=1.52, P=0.205)$. Solferino fue el sitio con el mayor porcentaje de germinación $(13.4 \pm 2.0 \%$; figura $5 \mathrm{a})$, mientras que Kantunilkin mostró el menor valor $(6.9 \pm 2.1 \%)$; el valor de Chiquilá fue intermedio (9.4 $\pm 1.44 \%)$. Dependiendo del sitio, la germinación de semillas inició a los 45 (Solferino), 90 (Chiquilá) y 105 (Kantunilkin) días después de la siembra.

El destino de las semillas no germinadas que permanecieron enterradas fue semejante en los tres sitios de estudio. Después de 6 meses, $48 \%$ de las semillas había desaparecido (figura 4a; ver cuadro 1 para una explicación 


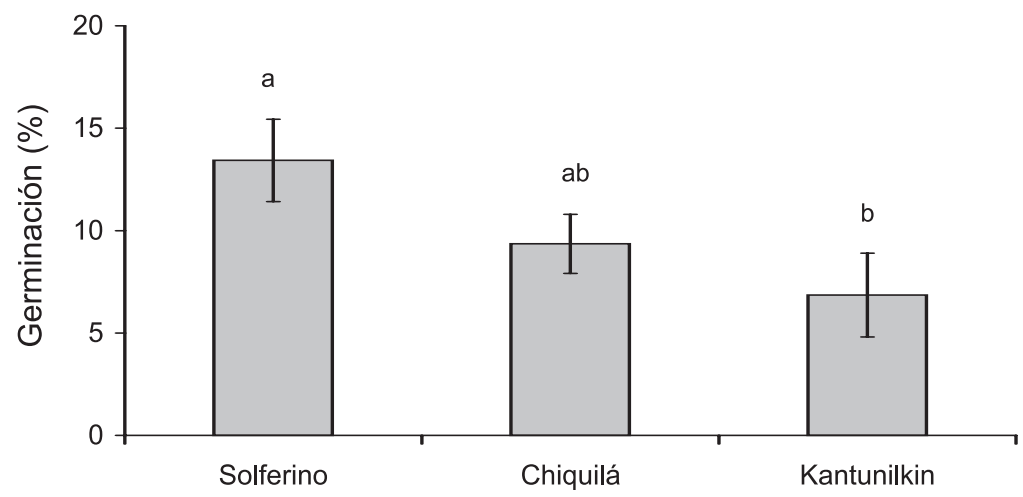

Figura 5. Variación espacial en la respuesta del porcentaje de germinación en semillas de Thrinax radiata. Las barras muestran los valores promedio y el error estándar. Con la misma letra se señalan los promedios que no mostraron diferencias estadísticamente significativas.

de las categorías de clasificación de las semillas). Este porcentaje se duplicó 12 y 18 meses después (figura $4 \mathrm{~b}, \mathrm{c}$ ). En el caso de las semillas clasificadas como muertas, el mayor porcentaje (10\%) se encontró 6 meses después de la siembra (figura 4a). Doce y 18 meses más tarde sólo fue posible asociar una causa de muerte precisa a 2 y $3 \%$ de las semillas iniciales (figura $3 b$ ), siendo el daño por insectos la principal causa de muerte. En otras ocasiones, las semillas clasificadas como muertas se encontraban fragmentadas y en estado de descomposición, y en otras el embrión se encontraba muerto, como reveló la prueba de TZ. De las semillas no germinadas, más de un tercio (35\%) permaneció viable después de 6 meses en el suelo (figura 4a). Sin embargo, 12 y 18 meses después el porcentaje de semillas viables fue despreciable ( 0 y $0.1 \%$ respectivamente; figura $4 b, c)$.

Las discrepancias observadas en los porcentajes asignados a los diferentes destinos de las semillas enterradas (por ejemplo: $0 \%$ viables a los 6 meses y $0.1 \%$ viables a los 18 meses) se deben a las variaciones existentes en las condiciones ambientales para los diferentes periodos en que permanecieron enterradas las semillas, y a que para cada periodo de tiempo (6, 12 y 18 meses) se desenterró y cuantificó

Cuadro 2. Coeficientes de correlación de Spearman $\left(\mathrm{r}_{\mathrm{s}}\right)$ entre las variables cobertura vegetal y porcentaje de germinación de semillas de Thrinax radiata. Se muestran los datos para los tres sitios y períodos de estudio.

\begin{tabular}{lccc}
\hline Sitio & \multicolumn{3}{c}{ Período (meses) } \\
& 6 & 12 & 18 \\
\hline Kantunilkin & 0.498 & -0.159 & 0.258 \\
Solferino & 0.268 & 0.006 & -0.390 \\
Chiquilá & -0.317 & -0.131 & 0.201 \\
\hline
\end{tabular}

de manera independiente un lote de 300 semillas.

El porcentaje de germinación de las semillas de chit en condiciones naturales no mostró una correlación significativa con la apertura del dosel por encima de las charolas. Los coeficientes de correlación de Spearman entre estas dos variables no resultaron significativos en ninguno de los casos analizados $(P \leq 0.05, \mathrm{~N}=10$; cuadro 2$)$.

\section{Discusión}

La inmadurez de las semillas de Thrinax radiata provocó una disminución y un retraso en la germinación, lo cual coincide con lo reportado para otras especies de palmas (Orozco-Segovia et al., 2003). Los altos porcentajes de germinación observados en el presente estudio en condiciones de oscuridad muestran que las semillas de Thrinax radiata no son fotoblásticas. Este resultado explica la ausencia de correlación entre la apertura del dosel y el porcentaje de germinación observado en el campo. La exposición de las semillas de Thrinax radiata a altas temperaturas tiene un efecto letal, como sucede para otras especies de palmas (Carpenter y Gilman, 1988; Orozco-Segovia et al., 2003).

La longevidad fisiológica de las semillas de Thrinax radiata fue relativamente corta. En el laboratorio la viabilidad de las semillas disminuyó considerablemente después de 6 meses de almacenamiento. La pérdida de viabilidad en semillas bajo condiciones de almacenamiento ha sido reportada para otras especies de palmas y se ha sugerido que la desecación del embrión es la causa de dicha pérdida (Broschat y Donselman, 1986; Orozco-Segovia et al., 2003).

El contenido de humedad de las semillas de Thrinax radiata presenta valores intermedios al compararlo con el de otras especies de palmas (Orozco-Segovia et al., 2003; Wood y Pritchard, 2003). Dado que no registramos los cambios en el contenido de humedad del embrión y el 
Cuadro 3. Respuesta de la germinación de semillas de Thrinax radiata en diferentes niveles de precipitación.

\begin{tabular}{lcccc}
\hline Sitio & Período & $\begin{array}{c}\text { Precipitación } \\
\text { acumulada } \\
\text { (mm) }\end{array}$ & $\begin{array}{c}\text { Inicio de la } \\
\text { germinación } \\
\text { (días) }\end{array}$ & $\begin{array}{c}50 \% \\
\text { germinación } \\
\text { (días) }\end{array}$ \\
\hline Kantunilkin & nov 98 - may 99 & 300 & 105 & 135 \\
& nov 99 - may 00 & 343 & 105 & 255 \\
\multirow{3}{*}{ Solferino } & nov 98 - may 99 & 452 & 45 & 90 \\
& nov 99 - may 00 & 256 & 135 & 270 \\
\hline
\end{tabular}

endospermo a lo largo del experimento de almacenamiento, no contamos con información suficiente para establecer la tolerancia a la desecación que presentan las semillas de esta especie de palma. La pérdida rápida de viabilidad bajo almacenamiento, ya sea en condiciones ambientales o a bajas temperaturas, no necesarimente indica que la semilla sea recalcitrante (Hong y Ellis, 1996). Es necesario investigar la supervivencia de las semillas bajo una gama más amplia de condiciones de almacenamiento para determinar el comportamiento preciso de Thrinax radiata (Hong y Ellis, 1996).

En el laboratorio, las semillas provenientes de frutos maduros germinaron en $15.5 \pm 1.1$ días, mientras que en el campo la germinación se inició entre 40 y 90 días después de la siembra. Este resultado, aunado al hecho de que ni la luz ni la escarificación incrementaron significativamente el porcentaje de germinación, sugiere que la disponibilidad de agua es el factor limitante de la germinación en condiciones naturales y no la impermeabilidad de la testa o la intolerancia a la sombra. Al comparar los registros de precipitación entre los dos sitios de estudio en los que existen estaciones meteorológicas (Kantunilkin y Solferino), se observa que la germinación de las semillas sucede primero en sitios y años con mayor precipitación (cuadro 3). Es necesario desarrollar un experimento en el que se manipule la disponibilidad de agua en condiciones naturales para probar esta hipótesis.

La longevidad ecológica de las semillas de Thrinax radiata fue relativamente corta, en concordancia con lo propuesto por Garwood (1989), y de acuerdo con los resultados del experimento de campo, Thrinax radiata presenta un banco de semillas estacional transitorio, compuesto por semillas con una longevidad de hasta 6 meses. Estos datos contrastan con las observaciones para una especie del mismo género, Thrinax morrissii, realizadas por Liu et al. (2004), quienes encontraron que el porcentaje de semillas viables después de 18 meses de enterramiento varía entre 30 y $60 \%$ dependiendo del tratamiento; al parecer, la disponibilidad de agua no es un factor limitante de la germinación de $T$. morrissii, pues a pesar de que durante el experimento las semillas permanecieron constantemente húmedas por el riego continuo, un porcentaje considerable de éstas no germinó. Sería interesante comparar los patrones de germinación de semillas de Thrinax radiata provenientes de individuos de vegetación costera, un hábitat semejante a aquel en el que se encuentra T. morrissii.

Aun cuando no fue posible asociar una causa particular a la pérdida de $90 \%$ de las semillas después de un año de enterradas, para el caso de la palma Astrocaryum murumuru Cintra (1997) muestra evidencias de una fuerte correlación entre las semillas removidas y la depredación de las mismas. Igualmente, diversos estudios reportan una fuerte depredación de semillas de palmas en condiciones naturales (Bradfrod y Smith, 1977; Smythe, 1989; Oyama, 1991; Harms y Dalling, 1995; Cintra y Horna, 1997). Al igual que para Thrinax radiata, la muerte de un elevado porcentaje de semillas (más de $40 \%$ ) debida al ataque por insectos ha sido reportada para Thrinax morrissii en Florida (Liu, 2004). La periodicidad de los registros utilizados en el presente estudio (cada 6 meses) no nos permitieron cuantificar en detalle la dinámica de depredación de las semillas de Thrinax radiata.

Si bien la depredación de semillas ha sido considerada como un factor determinante en la dinámica poblacional de plantas, especialmente en el caso de especies fugitivas (Harper, 1977; Louda, 1989), en un escenario de acelerada deforestación y fragmentación del hábitat, el efecto de la depredación de semillas también será un elemento importante en la dinámica poblacional de especies de vegetación madura. Por otro lado, en función de la rápida pérdida de viabilidad de las semillas observada bajo condiciones de almacenamiento, es probable que la baja humedad del suelo durante la época de sequía sea otro factor de muerte de las semillas en condiciones naturales.

El efecto negativo de la baja disponibilidad de agua sobre la germinacion y la supervivencia de las semillas de Thrinax radiata, así como la elevada depredación y la ausencia de un banco de semillas permanente, sugieren que el estableciemiento de nuevas poblaciones de Thrinax radiata depende fuertemente del mantenimiento de la vegetación madura. Además, es en estos parches de vegetación madura donde se encuentran los individuos reproductivos de esta especie (Olmsted y Álvarez-Buylla, 1995; Calvo-Irabién y Ceballos-González, 2004). En el área estudiada no se encontraron individuos de Thrinax radiata en parches de vegetación secundaria joven (5-15 años, L.M. CalvoIrabién, obs. pers.). Aunado a lo anterior, dado que la viabilidad y la germinación de las semillas se ven fuertemente influidas por las altas temperaturas, los fuegos, ya sean naturales o antropogénicos, reducirán cosiderablemente el potencial de regeneración de esta especie de palma.

Como se mencionó anteriormente, en la Península de Yucatán los troncos de Thrinax radiata han sido y siguen siendo utilizados para diferentes actividades de construc- 
ción (casas, palapas, trampas de langosta). Olmsted y Álvarez-Buylla (1995), con base en un estudio demográfico de esta especie realizado en la Reserva de Sian Ka'an, resaltaron que en general la cosecha de adultos tiene un efecto negativo en la tasa de crecimiento $(\lambda)$ de las poblaciones naturales. Por otro lado, dado que los cambios en la fecundidad de los adultos de esta palma presentan un impacto muy bajo sobre $\lambda$ (Olmsted y Álvarez-Buylla, 1995), y dado que el chit es una especie apreciada como planta ornamental (Durán et al., 2000), una alternativa de uso de esta especie es el cultivo de individuos en viveros comunitarios. En condiciones de vivero la germinación y la supervivencia de las plántulas son muy altas (ca. 90\%; Durán et al., 2000; Pérez, 2002), por lo que el cultivo de Thrinax radiata en viveros ejidales o comunitarios es una alternativa sencilla y poco costosa. Esta práctica no implica la muerte de los individuos adultos y por lo tanto su impacto sobre $\lambda$ será comparativamente menor que la cosecha de troncos. Adicionalmente, dado que el chit es una especie longeva, un adulto reproductivo produce semillas durante 40 ó 60 años de su vida. Como parte del plan de manejo de esta palma se han establecido tres viveros comunitarios donde se propagan exitosamente individuos de la palma chit (Calvo-Irabién y Macías-Cuellar, 2000; Calvo-Irabién y Ceballos-González, 2004). Sin embargo, la colecta de semillas deberá hacerse bajo un constante y cuidadoso monitoreo. Se deberá poner especial atención a los métodos de cosecha, pues en ocasiones se derriba la palma adulta para tener acceso a las semillas (L.M. CalvoIrabién, obs. pers). Asimismo, es recomendable que las semillas cosechadas provengan de individuos diferentes para tener una mejor representación de la diversidad genética de la especie. Posiblemente la única desventaja de esta estrategia es que, dada la lenta tasa de crecimiento de los individuos de esta especie (Olmsted y Álvarez-Buylla, 1995; Pérez, 2002), los individuos cultivados en vivero sólo alcanzan tallas comerciales 2 ó 3 años después de la siembra (Durán et al., 2000).

En conclusión, dado que nuestros resultados sugieren que la conservación ex situ de Thrinax radiata, en colecciones de semillas, no es una alternativa viable, proponemos para su conservación una estrategia que combine la conservación de su hábitat con la propagación en viveros de propiedad comunal, como un uso alternativo al corte de troncos.

\section{Agradecimientos}

Agradecemos la revisión y comentarios de Armando Escamilla (q.e.p.d.), Eric Graham y Hugh Harries, así como de la Dra. Alma Orozco y un árbitro anónimo por sus atinados comentarios durante la revisión del manuscrito. También agradecemos la ayuda en el campo de Edward Pérez, Don Tomás Canul y Don Edilberto Chi Tah, y de
Luis Simá y Gabriel Dzib en el trabajo de laboratorio. Un agradecimiento especial al Jardín Botánico Regional del CICY por las facilidades proporcionadas. Este proyecto fue parcialmente financiado por la Comisión Nacional para el Conocimiento y Uso de la Biodiversidad, el Fondo Mexicano para la Conservacion de la Naturaleza y el programa SISIERRA-CONACYT.

\section{Literatura citada}

Almanza H. 2000. Percepciones locales de la naturaleza en el Área de Protección de Flora y Fauna "Yum Balam" en Quintana Roo. Tesis de Licenciatura, Facultad de Antropología, Universidad Autónoma de Yucatán, Mérida, 155 pp.

Balick M.J. y Beck H.T. 1990. Useful Palms of the World. A Synoptic Bibliography. Columbia University Press, Nueva York.

Bewley J.D. y Black M. 1994. Seeds: Physiology of Development and Germination. Plenum Press, Nueva York.

Bradford D.F. y Smith C.C. 1977. Seed predation and seed number in Scheelea palm fruits. Ecology 58:667-673.

Broschat T.K. y Donselman H. 1986. Factors affecting storage and germination of Chrysalidocarpus lutescens seeds. Journal of the American Society of Horticulture Science 111:872-877.

Broschat T.K. y Donselman H. 1987. Effects of fruit maturity, storage, pre-soaking and seed cleaning on germination in three species of palms. Journal of Environmental Horticulture 5:6-9.

Brown K.E. 1976. Ecological studies of the cabbage palm, Sabal palmetto. III. Seed germination and seedling establishment. Principes 20:98-115.

Cabrera E., Sousa M., Téllez O. y López A. 1982. Imágenes de la Flora Quintanarroense. Centro de Investigaciones de Quintana Roo, A.C., México, D.F.

Calvo-Irabién L.M. y Ceballos-González G. 2004. La palma Chit, historia del desarrollo de un plan de manejo para una especie amenazada. En: Armijo N. y Llorens C. Eds. Uso, Conservación y Cambio en los Bosques de Quintana Roo, pp. 179-202, Universidad de Quintana Roo y CONACYT, Chetumal.

Calvo-Irabién L.M. y Macías-Cuellar H. 2000. El Cultivo de Chit: Manual para su Propagación en Viveros. Centro de Investigación Científica de Yucatán y Comisión Nacional para el Conocimiento y Uso de la Biodiversidad, Mérida.

Calvo-Irabién L.M., Olmsted I., Durán R., Macías-Cuellar H. y Almanza H. 1998. Programa de Manejo Forestal para la Palma Thrinax radiata (Chit), en los Ejidos de Kantunilkin, Solferino y Chiquilá-San Ángel, Quintana Roo. Centro de Investigación Científica de Yucatan, Mérida.

Carpenter W.J. y Gilman E.F. 1988. Effect of temperature and desiccation on the germination of Thrinax morrisii. Proceedings of the Florida State Horticulture Society 101:288-290.

Cintra R. 1997. Leaf litter effects on seed and seedling predation of the palm Astrocaryum murumuru and the legume tree Dypterix micrantha in Amazonian forest. Journal of Tropical Ecology 13:709-725.

Cintra R. y Horna V. 1997. Seed and seedling survival of the palm Astrocaryum murumuru and the legume tree Dipteryx micran- 
tha in gaps in Amazonian forest. Journal of Tropical Ecology 13:257-277.

Cortina-Villar S., Mendoza M., Ogneva P.M. y Himmelberger Y. 1999. Cambios en el uso del suelo y deforestación en el sur de los estados de Campeche y Quintana Roo, México. Boletín del Instituto de Geografía de la UNAM. Investigaciones Geográficas 38:41-56.

DOF [Diario Oficial de la Federación]. 1994. Decreto por el que se declara como área natural protegida, con carácter de área de protección de flora y fauna, la región conocida como Yum Balam, ubicada en el Municipio de Lázaro Cárdenas, Estado de Quintana Roo, 6 de junio de 1994.

DOF [Diario Oficial de la Federación]. 2002. Norma Oficial Mexicana Nom-059-Ecol-2001, Protección Ambiental-Especies Nativas de Mexico de Flora y Fauna Silvestres-Categorías de Riesgo y Especificaciones para su Inclusión, Exclusión o Cambio - Lista de Especies en Riesgo, 6 de marzo de 2002, pp. 2-56.

Durán R., Dorantes A., Simá P. y Méndez M. 2000. Manual de Propagación de Plantas Nativas de la Península de Yucatán. Vol. 2. Centro de Investigación Científica de Yucatán, Mérida. Fenner M. 1985. Seed Ecology. Chapman and Hall, Londres.

Flores J.S. 2001. Etnoflora Yucatanense. Florística, Etnobotánica y Ecología. Universidad Autónoma de Yucatán. Mérida.

Flores J.S. y Espejel I. 1994. Tipos de Vegetación de la Península de Yucatán. Universidad Autónoma de Yucatán, Mérida.

Garwood N.C. 1989. Tropical soil seed banks: a review. En: Leck A., Parker V.T. y Simpson R.L. Eds. Ecology of Soil Seed Banks, pp. 149-209, Academic Press, San Diego.

Gentry A.H. 1990. Four Neotropical Rainforests. Yale University Press, New Haven.

Harms K.E. y Dalling J.W. 1995. Observations on the seasonal consistency in germination timing for Scheelea zonensis. Principes 39:104-106.

Harper J.L. 1977. Population Biology of Plants. Academic Press, Londres.

Hong T.D. y Ellis R.H. 1996. A protocol to determine seed storage behavior. En: Engels J.M.M. y Tool J. Eds. IPGRI. Technical Bulletin No. 1. International Plant Genetic Resources Institute. Roma.

ISTA (International Seed Testing Association). 1999. International Rules for Seed Testing. Seed Science and Technology 27 (Supplement): 1-333.

La Torre-Cuadros M.A. e Islebe G.A. 2003. Traditional ecological knowledge and use of vegetation in southeastern Mexico: a case study from Solferino, Quintana Roo. Biodiversity and Conservation 12:2455-2476.

Lazcano M., Vázquez-Sánchez M., March I., Núñez H. y Fuller M. 1995. La Región de Yalahau: Propuesta para el Establecimiento de una Zona de Conservación y Desarrollo Sostenible en el Norte de Quintana Roo. CECRN, Colegio de la Frontera Sur, Chetumal.

Lemmon P.E. 1956. A spherical densiometer for estimating forest overstory density. Forest Science 2:314:320.

Liu H., Platt S.G. y Borg C.K. 2004. Seed dispersal by the Florida box turtle (Terrapene carolina bauri) in pine rockland forest of the lower Florida Keys, United States. Oecologia 138:539546.

Louda S.M. 1989. Predation in the dynamics of seed regeneration. En: Leck A., Parker V.T. y Simpson R.L. Eds. Ecology of Soil
Seed Banks, pp. 25-51, Academic Press, San Diego.

Marcus J. y Banks K. 1999. A practical guide to germinating palm seeds. Palms 43:56-59.

Meerow A.W. 2000. Palm Seed Germination. University of Florida IFAS Cooperative Bulletin 274:1-10

Moreno E. 1996. Análisis Físico y Biológico de Semillas Agrícolas. Instituto de Biología, Universidad Autónoma de México, México, D.F.

Murdoch A.J. y Ellis R.H. 1992. Longevity, viability and dormancy. En: Fenner M. Ed. Seeds: the Ecology of Regeneration in Plant Communities, pp. 193-229, CAB International, Londres.

Olmsted I. y Álvarez-Buylla E. 1995. Sustainable harvesting of tropical trees: Demography and matrix models of two palm species in Mexico. Ecological Applications 5:484-500.

Olmsted I., Durán R., González-Iturbe J.A., Calvo-Irabién L.M., Remolina F. y Canela J. 2000. Programa de Manejo del Área de Protección de Flora y Fauna Silvestre Yum Balam. Centro de Investigación Científica de Yucatán, Mérida.

Orellana R., Balam M., Bañuelos I., García E., González-Iturbe J.A., Herrera F. y Vidal J. 1999. Evaluación climática. En: Chico-Ponce de León F. y García de Fuentes A. Eds. Atlas de Procesos Territoriales de Yucatán, pp. 163-175, Universidad Autónoma de Yucatán, Mérida.

Orellana R. y Durán R. 1992. Las palmas de la Península de Yucatán: un patrimonio que debemos conservar. Gaceta Universitaria 14:22-28.

Orozco-Segovia A., Batis A., Rojas-Aréchiga M. y Mendoza A. 2003. Seed biology of palms: a review. Palms 47:79-94.

Oyama K. 1991. Seed predation by a curculionid beetle on the dioecious palm Chamaedorea tepejilote. Principes 35:156160.

Pérez E. 2002. Germinación y crecimiento de la palma Thrinax radiata Lodd. ex Schult. et Schult.f. en condiciones naturales y controladas. Tesis de Licenciatura, Universidad Autónoma de Yucatán, Faculta de Medicina Veterinaria y Zootecnia. Mérida, $77 \mathrm{pp}$.

Quero H.J. 1992a. Current status of Mexican palms. Principes 36:203-216.

Quero H.J. 1992b. Las Palmas Silvestres de la Península de Yucatán. Instituto de Biología, Universidad Autónoma de México, México, D.F.

Roys R.L. 1931. The Ethno-Botany of the Maya. The Tulane University of Louisiana. Nueva Orleans.

Sánchez-Sánchez O. e Islebe G.A. 2002. Tropical forest communities in southeastern Mexico. Plant Ecology 158:183-200.

Scariot A. 1999. Forest fragmentation effects on palm diversity in central Amazonia. Journal of Ecology 87:66-76.

Schemske D., Husband W.B.C., Ruckelshaus M.H., Goodwillie C., Parker I.M. y Bishop J.G. 1994. Evaluating approaches to the conservation of rare and endangered plants. Ecology 75:584-606.

Smythe N. 1989. Seed survival in the palm Astrocaryum standleyanum: evidence for dependence upon its seed dispersers. Biotropica 21:50-56.

Sokal R.R. y Rohlf F.J. 1995. Biometry: The Principles and Practice of Statistics in Biological Research. W.H. Freeman, Nueva York.

Standley P.C. 1930. Flora of Yucatan. Publications - Field Museum of Natural History. Botanical Series 3:157-492.

Terborgh J. 1986. Keystone plant resources in the tropical forest. 
En: Soulé M.E. Ed. Conservation Biology: The Science of Scarcity and Diversity, pp. 330-344, Sinauer, Sunderland.

Turner B.L. II, Cortina-Villar S., Foster D., Geoghegan J., Keys E., Klepeis P., Lawrence D., Macario-Mendoza P., Manson S., Ogneva-Himmelberger A., Plotkin A.B., Pérez-Salicrup D., Chowdhury R.R., Savitsky B., Schneider L., Schmook B. y Vance C. 2001. Deforestation in the southern Yucatán peninsular region: an integrative approach. Forest Ecology and Management 154:353-370.

Fecha de recepción: 16 de junio de 2005

Versión corregida: 29 de septiembre de 2005

Aceptado: 11 de octubre de 2005
Vázquez-Yanes C. y Orozco-Segovia A. 1993. Patterns of seed longevity and germination in the tropical rainforest. Annual Review of Ecology and Systematics 24:69-87.

Wood C.B. y Pritchard H.W. 2003. Germination characteristics of fresh and dried Hyophorbe lagenicaulis seeds. Palms 47:4550 .

Yacubson D. 1980. Principios Fundamentales de los Seres Vivos. Reunión sobre problemas en semillas forestales tropicales. Tomo 1. INIFAP, México, D.F. 